\title{
Self-regulation of spasm and spasticity in cerebral palsy
}

\author{
PETER D NEILSON, JANET MCCAUGHEY \\ From the Spastic Centre Research Unit, Department of Neurology, The Prince Henry Hospital and School \\ of Medicine, University of New South Wales, Australia
}

SUMMARY Four young adult cerebral palsied subjects with a mixture of spasticity and athetosis attended an experimental reflex training program for three one-hour sessions each week over an 18 month period. During each session on-line measures of contraction level and tonic stretch reflex sensitivity from the biceps brachii muscle were shown to the subject on meter displays. Subjects were asked to attempt to control the displays. They were given goals such as: (1) reduce both contraction level and reflex sensitivity displays to zero and (2) increase the contraction level display to $10 \%$ of maximum while keeping the reflex sensitivity display at a minimum. Achievement of goals was automatically sensed and used to activate a cassette tape of the subject's favourite music. Contraction level and reflex sensitivity scores were averaged over one-minute intervals to provide a record of long term progress. Elbow-angle and IEMG data were recorded on FM tape for off-line analysis. All four subjects learned to suppress involuntary muscle activity and resting tonic stretch reflex responses. They also learned to produce a two or three-fold variation in action tonic stretch reflex sensitivity while sustaining $10 \%$ maximum voluntary contraction. In other words, subjects learned to self-regulate spasm and spasticity at the elbow and to regulate tonic stretch reflex sensitivity independently of contraction level. A visual tracking task requiring voluntary movement about the elbow was employed to assess improvement in functional control of elbow movement. One athetotic subject improved tracking accuracy as a consequence of reducing the amount of involuntary arm movement while the other three subjects showed negligible improvement in functional control.

Cerebral palsy implies non-progressive brain damage, sustained before, during or shortly after birth, which impairs control of movement. ${ }^{1}$ Hypertonicity and spasm are symptoms of cerebral palsy which can disrupt control of movement and can lead to deformities of bones, joints and muscles. ${ }^{2}$ Such deformities often require surgical correction such as, for example, the recession of the origin of gastrocnemius-soleus muscle or the lengthening or even division of the tendo Achillis to enable the patient to lower his heels to the floor. ${ }^{2}$ Unless spasm and muscle tone are reduced in conjunction with such corrective surgery, there can be no guarantee that contractures and/or deformities will not recur. Clearly, techniques for reduction of spasm and

Address for reprint requests: Dr PD Neilson, Spastic Centre Research Unit, The Prince Henry Hospital, Little Bay, Sydney, NSW 2036, Australia

Received 1 October 1981

Accepted 18 November 1981 muscle tone are an important component of therapy for cerebral palsy.

But what is muscle tone and how can it be reduced? Muscle tone is assessed clinically by asking the patient to relax and passively moving the limb through a range of movement about the joint while experiencing the resistance to movement. Hypertonicity is experienced as an increased resistance to movement and in cerebral palsy this is referred to as rigido-spasticity since individual patients often have a mixture of spasticity and rigidity. Rigido-spasticity is due to hypersensitivity of tonic stretch reflex pathways. ${ }^{2-4}$ Many cerebral palsied individuals are able to relax and the relaxed limb can be moved passively without evoking any tonic stretch reflex response. According to the clinical method of assessing muscle tone described above, such patients have normal muscle tone when they are at rest. Most cerebral palsied individuals, however, do have tonic stretch reflex responses to passive stretching when they are at rest and are described clinically as 
having rigidity or spasticity or both. It has been shown that as soon as a cerebral palsied individual voluntarily contracts muscles, tonic stretch reflex pathways are functionally reorganised and the magnitude, timing and waveform of the responses are completely different from those measured in the same cerebral palsied subject at rest. ${ }^{4}$ In other words, rigido-spasticity assessed by a clinician with the subject at rest is different from the rigido-spasticity experienced by the cerebral palsied individual himself during voluntary movement of the same limb. Since rigido-spasticity is thought to disrupt voluntary movement in cerebral palsy, it is the abnormal tonic stretch reflex during voluntary activity which should be assessed and controlled. To differentiate between tonic stretch reflex responses measured with the subject at rest and those measured during voluntary activity, the terms "resting tonic stretch reflex" and "action tonic stretch reflex" have been proposed. 4

It has been shown that normal subjects can be trained to self-regulate action tonic stretch reflex sensitivity independently of contraction level. ${ }^{4}$ By displaying on-line measures of contraction level and action tonic stretch reflex sensitivity to normal subjects, they can be trained to produce a four-fold variation of action tonic stretch reflex sensitivity on demand, while maintaining a constant cocontraction at $30-50 \%$ of maximum. Moreover, when the action tonic stretch reflex sensitivity is low, the arm feels loose to the experimenter despite the co-contraction of elbow muscles. This raises the important question, can cerebral palsied subjects be trained to self-regulate rigido-spasticity? This report describes an 18 month action reflex training programme in which a "spasticity meter" was employed to provide on-line measures of "contraction level" and "reflex sensitivity" to four young adult cerebral palsied subjects. The aim of the study was to determine whether or not cerebral palsied individuals can be trained to self-regulate spasm and spasticity at the elbow.

\section{Methods}

The subject sat in a wheel chair with his right upper-arm resting on a table at his side and with his forearm vertical. The arm was strapped into an arm-frame which restrained movement to flexion-extension about the elbow (fig 1). A potentiometer attached to the arm-frame recorded elbow-angle. Surface electrodes were attached over the belly of the biceps brachii muscle and the electromyogram (EMG) activity was amplified in a Dynamic Electronics 3160 EMG preamplifier (gain $=1000$, bandwidth $=80$ $2500 \mathrm{~Hz}$ ) and displayed on an oscilloscope. The EMG was full-wave rectified and low-pass filtered by a 2 nd order filter $(\mathrm{T} 1=\mathrm{T} 2=0.03 \mathrm{~s})$ in an EAI 180 analog computer to provide what will be referred to as the IEMG

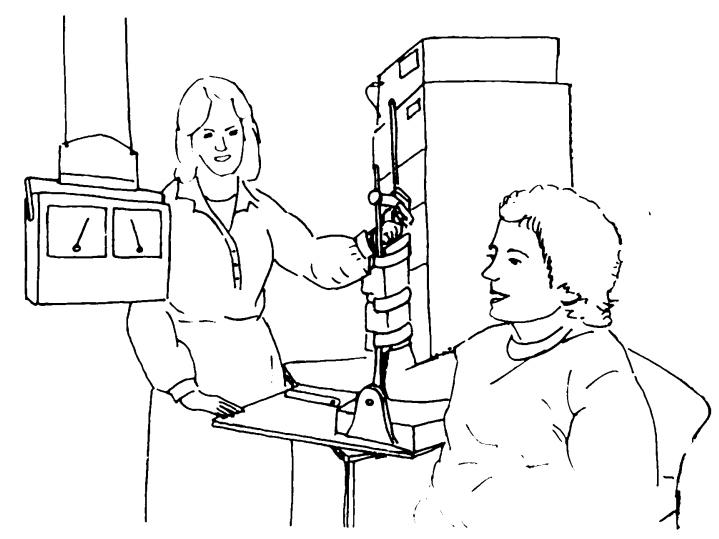

Fig 1 The training situation showing subject sitting in wheel chair with right arm strapped into arm-frame. The subject attempts to control two meters displaying contraction level and reflex sensitivity while the experimenter passively oscillates the arm back and forth through 5 or 10 degrees at the elbow.

signal. The elbow-angle and IEMG signals were recorded on FM tape for subsequent transcription to a model 5 Grass polygraph.

A "spasticity meter" which provided on-line displays of "contraction level" and "reflex sensitivity" was also patched on the EAI 180 analog computer. The experimenter oscillated the arm back and forth through 5 degrees about the elbow and the elbow-angle and EMG signals were amplified and fed to the spasticity meter. The contraction level display was produced as follows: the full-wave rectified EMG was smoothed in a long time constant ( $T=2.0 \mathrm{~s}$ ) 1st order low-pass filter and displayed on a $15 \mathrm{~cm}$ meter. The gain of the EMG amplifier was adjusted so that full scale deflection on the meter corresponded to $33 \%$ of maximum contraction. The reflex sensitivity display was produced as follows: the elbow-angle and IEMG signals were connected to "sample and hold" circuits which computed the maximum peak to peak value of each signal during every $0.5 \mathrm{~s}$ interval. These voltages were connected to a divider circuit which computed the ratio of peak to peak IEMG variation to peak to peak elbow-angle variation during each $0.5 \mathrm{~s}$ interval. The output voltage from the divider circuit was updated every $0.5 \mathrm{~s}$ and was proportional to the reflex sensitivity (that is, ratio of change in IEMG to change in elbow-angle). This voltage was smoothed in a long time constant $(\mathrm{T}=2 \cdot 0 \mathrm{~s}) 1 \mathrm{st}$ order low-pass filter and displayed on a second $15 \mathrm{~cm}$ meter. The reflex sensitivity meter was calibrated from 0 to 10 and the gain of the meter was adjusted so that it displayed about 8 for the largest reflex response from each subject.

Apart from on-line displays of contraction level and reflex sensitivity, two other features were incorporated in the spasticity meter. Threshold detector circuits were included so that threshold levels could be set on both contraction level and reflex sensitivity displays. If the subject improved performance so that the meter readings 
crossed the threshold settings (for example, contraction level exceeded the threshold setting on the contraction level meter and reflex sensitivity reduced below the threshold setting on the reflex sensitivity meter), the improvement was detected and a cassette recorder playing the subject's favourite music was automatically switched on. Conversely, if performance deteriorated and the displays crossed back over the threshold settings, the music was automatically turned off. The threshold settings could be varied from day to day so that a slightly improved performance was required to achieve the music reward. One minute timer and averager circuits were incorporated in the spasticity meter so the average readings of the contraction level meter and the reflex sensitivity meter over a one minute interval, initiated by the push of a button, could be computed and displayed on a digital meter. These one-minute-average readings were graphed to provide a record of long-term progress.

Four young adult cerebral palsied subjects, three female and one male, aged 24-29 years, were involved in the reflex training programme. All four subjects were severely disabled with a mixture of spasticity and athetosis, although two were predominantly athetotic and two were predominantly spastic. The athetotic subjects had negligible arm function and often had their arms restrained to control involuntary arm movements. Arm movements of the spastic subjects were slow and spasticity was clearly present at the elbow on clinical examination. Each subject attended the laboratory for three 1.0 hour sessions per week throughout the 18 month programme.

The first three months of the programme were used to obtain baseline scores. With the right arm strapped into the arm-frame and contraction level displayed, subjects were asked to: (i) reduce muscle activity to zero, (ii) reduce muscle activity to zero and maintain a loose arm while the experimenter passively oscillated the arm back and forth about the elbow, (iii) maintain a constant level of contraction and stiffen the arm to resist passive movement about the elbow and, (iv) maintain a constant level of contraction and loosen the arm so as not to resist passive movement about the elbow. During this baseline period no reflex training was given to the subjects. Reflex sensitivity measures were not displayed to the subjects, although one-minute-average contraction level and reflex sensitivity scores were recorded. Subjects were asked "to reduce muscle activity to zero" rather than "to relax", because it was found that, to a cerebral palsied individual, being relaxed does not necessarily mean reducing muscle activity to zero. Being relaxed implies a state of no effort. A conscious effort is often required to inhibit involuntary muscle activity and so the subject does not necessarily perceive a state of zero muscle activity as a state of relaxation.

Subjects performed a visual pursuit tracking test during the baseline recording period and again on completion of the reflex training programme. The tracking test provided a measure of the subject's functional control of elbow movement. The elbow-angle signal from the goniometer attached to the arm-frame was used to control the vertical displacement of a response marker on a $43 \mathrm{~cm}$ graphics display screen. A stimulus marker moved vertically in an irregular fashion on the same screen driven by a pre- recorded stimulus signal with a duration of one minute. The one minute duration tracking test was performed twice by each subject with a 45 minute rest between each test. The scores presented below were averaged across these two tests. Movements of the stimulus marker were slow so that the tracking task was relatively easy, at least for a normal subject. A power spectrum of the stimulus marker movements showed maximum variance at $0.1 \mathrm{~Hz}$, decreasing to negligible levels by $0.8 \mathrm{~Hz}$. The task for the subject was to move the arm back and forth through 20 degrees at the elbow about the 90 degree position so as to keep the response marker aligned with the moving stimulus marker. Movements of stimulus and response markers were sampled synchronously at a rate of 10 samples/s and stored in computer files for subsequent analysis. The response signal was subtracted from the stimulus signal to provide a measure of tracking error and the root mean square (RMS) value of the error signal was computed to provide a measure of overall tracking performance. The relative RMS error score was obtained by dividing the RMS error score by the RMS value of the stimulus signal and expressing the ratio as a percentage. A cross correlational analysis between the stimulus and the response signals provided a detailed measure of tracking performance. ${ }^{5}$ Variance of response movement was computed for each test and separated into two components: (i) variance of response movement correlated with stimulus marker movement and (ii) variance of response movement not correlated with stimulus marker movement. The latter provided a measure of the amount of inappropriate arm movement made by the subject during each test. ${ }^{5}$ The ratio of the variance of correlated response movement to the variance of stimulus marker movement was computed for each test. The square root of this ratio is referred to as "overall gain" and provides a measure of the amount of appropriate response movement produced by each subject relative to the amount of stimulus marker movement. For perfect tracking, overall gain has a value of unity.

After completion of the 3 month baseline recording period, subjects commenced the reflex training programme. The displays were arranged so that subjects could monitor their contraction level and reflex sensitivity measures (fig 1). Music reinforcement was provided for improvement in ability to achieve the set goals and graphs of one-minute-average contraction level and reflex sensitivity scores were displayed so that subjects could monitor their own long term progress. The following sequence of events occurred during each training session. (1) After the arm was strapped into the arm-frame, electrodes applied and signal quality checked, subjects were asked to produce a maximum co-contraction of elbow flexor and extensor muscles. (2) Subjects were asked to produce a maximum contraction of biceps brachii muscle by pulling against the experimenter. Gain of the EMG amplifier was adjusted so that full scale deflection on the contraction level meter corresponded to $33 \%$ of maximum. This calibration procedure enabled all subsequent EMG measures to be expressed as a percentage of maximum. (3) With the arm-frame supported, subjects were asked to try to reduce the contraction level display to zero while a one-minute-average contraction 
level score was obtained. (4) Subjects were asked to try to reduce both the contraction level and the reflex sensitivity displays to zero while the arm was passively oscillated back and forth through 5 degrees at the elbow by the experimenter. The experimenter monitored elbow-angle displacement on an oscilloscope, carefully controlling both amplitude and frequency of the applied sinusoidal displacement. (5) Subjects were asked to try to maintain a constant contraction level at $10 \%$ of maximum by cocontracting elbow flexor and extensor muscles and at the same time to try to increase reflex sensitivity as much as possible while the experimenter passively oscillated the arm back and forth about the elbow. One-minute-average contraction level and reflex sensitivity scores were obtained. (6) Subjects were asked to try to slowly increase contraction level to $10 \%$ of maximum by co-contracting elbow flexor and extensor muscles and at the same time to try to keep the reflex sensitivity at a minimum while the experimenter passively oscillated the arm back and forth about the elbow. After the $10 \%$ contraction level was achieved, oneminute-average contraction level and reflex sensitivity scores were obtained.

Typically, about $80 \%$ of the training time during each session was given to regulation of action tonic stretch reflex sensitivity. The best one-minute-average contraction level and reflex sensitivity scores for each subject in each task in each training session were graphed.

(a) ATH SUBJ 1
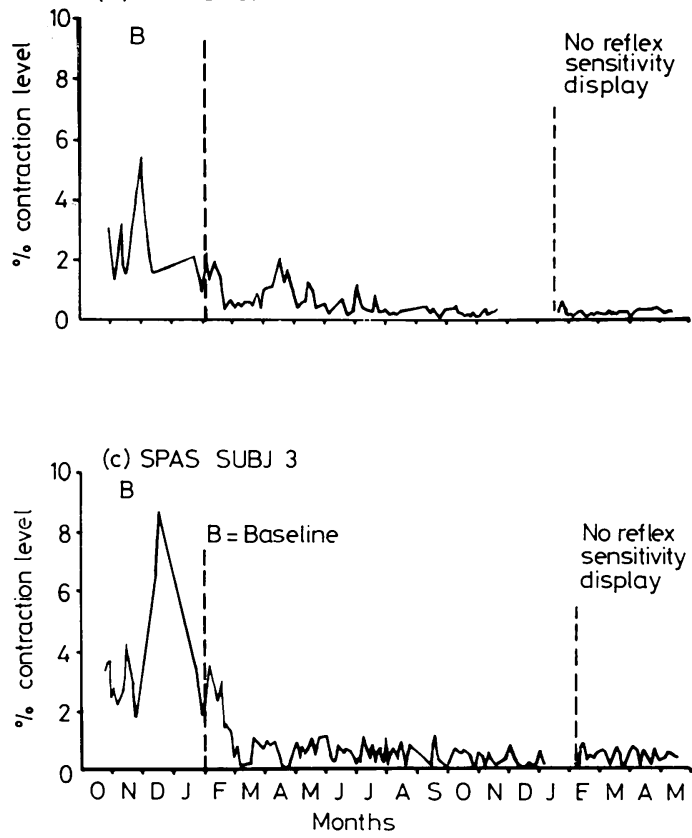

Results

Initially, all four subjects experienced difficulty in reducing biceps brachii muscle activity to zero. There was sustained involuntary background activity which fluctuated between $0-20 \%$ of maximum. There were also occasional vigorous spasms. The magnitude of the involuntary activity and the frequency of spasms varied from day to day, so, in terms of their ability to suppress involuntary activity, all subjects had "good days" and "bad days". Ability to suppress involuntary muscle activity improved during the reflex training period as shown by the graphs of one-minute-average contraction level scores plotted against days of training (fig 2). After training, all subjects reported that relaxation or a state of zero effort corresponded to either zero or a low contraction level and considerable effort was required to maintain $10 \%$ contraction.

Before training, when subjects were asked to try to reduce contraction level to zero while the arm was passively oscillated back and forth about the elbow, resting tonic stretch reflex responses were clearly

(b) ATH SUBJ 2

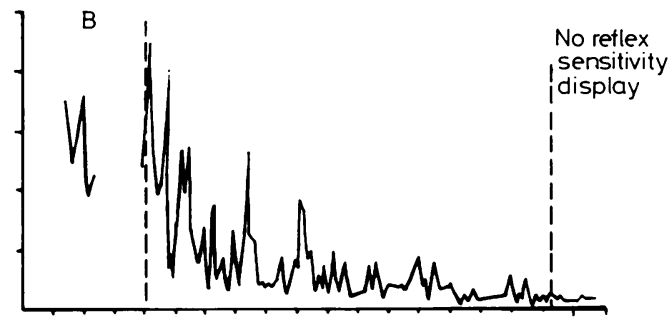

(d) SPAS SUBJ 4

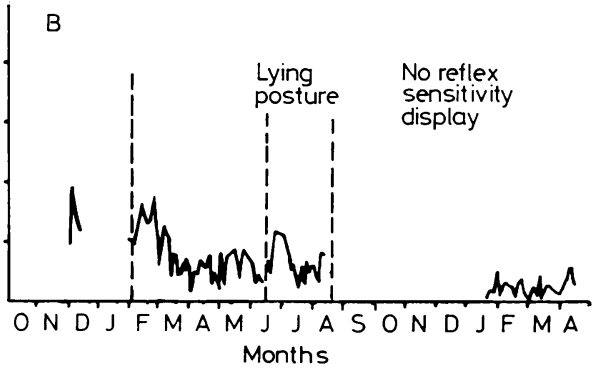

Fig 2 Graphs of one-minute-average contraction level scores expressed as a percentage of a maximum contraction versus number of days of training. Subject was attempting to reduce the contraction level of biceps brachii to zero. (a) athetotic subject 1, (b) athetotic subject 2, (c) spastic subject 3 , (d) spastic subject 4 showing effect of change from a sitting to a lying posture. 
SPAS SUBJ 3 Before reflex training

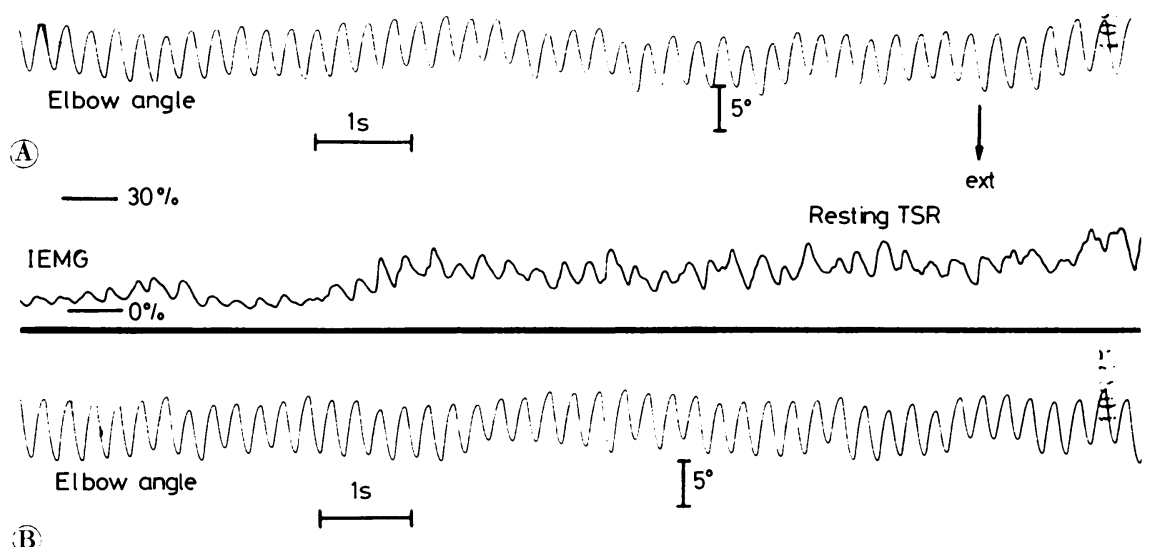

(B)

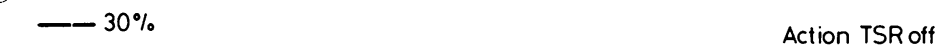

WhG

(C)

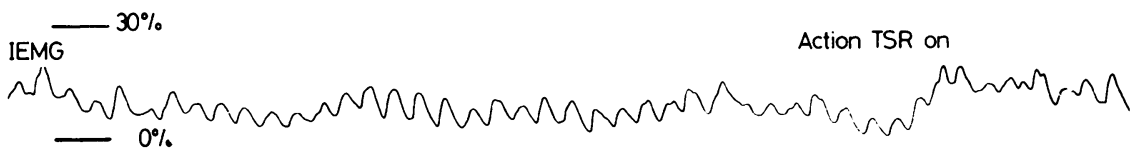

Fig 3 Sections of polygraph tracing from spastic subject 3 showing elbow-angle and IEMG signals recorded before reflex training (a) while subject was attempting to reduce contraction level and reflex sensitivity to zero (resting tonic stretch reflex); (b) while subject was voluntarily maintaining a constant contraction level at $10 \%$ of maximum and trying to reduce reflex sensitivity (action tonic stretch reflex off); (c) while subject was voluntarily maintaining a constant contraction level at $10 \%$ of maximum and trying to increase reflex sensitivity (action tonic stretch reflex on). Notice (i) fluctuating level of involuntary background activity in resting tonic stretch reflex recording and corresponding variation in magnitude of resting tonic stretch reflex response; (ii) lack of control over action tonic stretch reflex sensitivity; (iii) similarity in magnitude and waveform between resting and action tonic stretch reflex responses. Calibration bars represent $0 \%$ and $30 \%$ of maximum contraction.

present in the IEMG (fig 3a). There was also a fluctuating level of background involuntary activity (fig 3a). The magnitude of the resting tonic stretch reflex response increased with the level of involuntary background activity (fig 3a). The magnitude and waveform of the resting tonic stretch reflex response with a given level of involuntary background activity was similar to the magnitude and waveform of the action tonic stretch reflex response with the same level of sustained voluntary activity (fig 3 ).

During the reflex training period all subjects learned to suppress resting tonic stretch reflex re- sponses (fig 4). Improvement occurred most rapidly during the first three months but then improved gradually until the magnitude of the resting tonic stretch reflex response was reduced to a negligible size (fig 5). The magnitude of the resting tonic stretch reflex response and the level of involuntary background activity decreased together (fig 4 , cf figs 2 and 5).

Initially, when subjects sustained a voluntary contraction at $10 \%$ of maximum, they had no control over action tonic stretch reflex sensitivity independently of contraction level (fig 6, before 

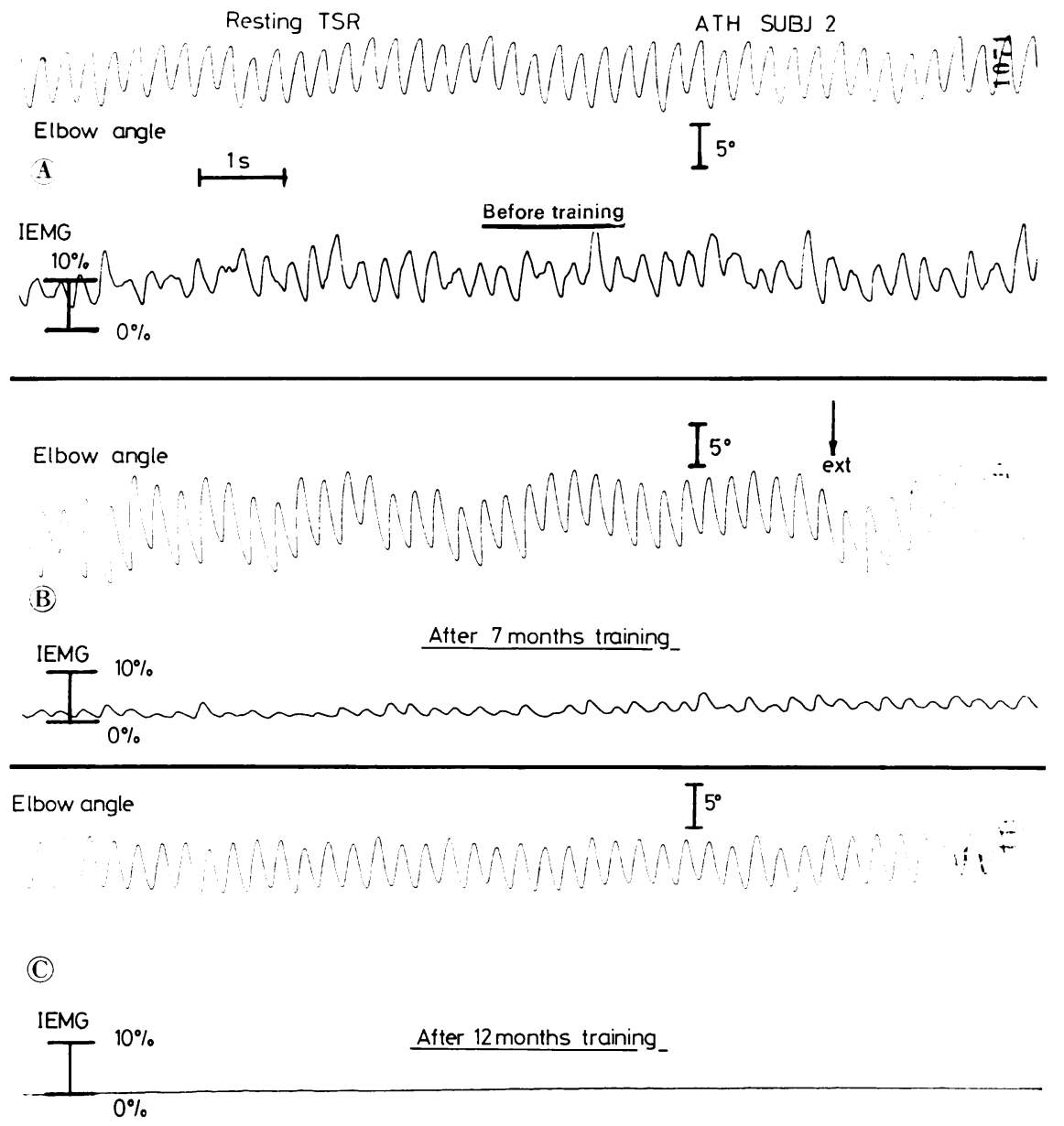

Fig 4 Sections of polygraph tracings of elbow-angle and IEMG signals from athetotic subject 2 showing resting tonic stretch reflex responses (a) before reflex training; (b) after 7 months of reflex training; (c) after 12 months of reflex training. Notice that involuntary background activity and magnitude of resting tonic stretch reflex response reduce together. Calibration bars represent $0 \%$ and $10 \%$ of maximum contraction.

training). During the reflex training period subjects gradually acquired the ability to regulate action tonic stretch reflex sensitivity two or three-fold while maintaining a constant contraction at $10 \%$ of maximum (fig 6, after training). The time course for each subject to acquire ability to regulate action tonic stretch reflex sensitivity is shown in fig 7 .

Two additional tests were made on spastic subject 4. (1) After four months training the subject was changed from a sitting to a lying posture and ability to suppress involuntary activity, reduce resting tonic stretch reflex responses and regulate action tonic stretch reflex sensitivity independently of contraction level, were all impaired by this change in posture (figs $2 \mathrm{~d}, 5 \mathrm{~d}, 7 \mathrm{~d}$ ). Training then continued for two months with the subject in a lying posture and control of all of these variables gradually improved (figs 2d, 5d, 7d). (2) At the end of this two month training, ability to transfer the skill learned on the right arm to the untrained left arm was assessed. Without being shown the reflex sensitivity display, the subject was asked to increase and decrease action tonic stretch reflex sensitivity in the left arm biceps brachii muscle while sustaining $10 \%$ of maximum contraction. Polygraph tracings (fig 8) show that the subject was able to regulate action 
(a) ATH SUBJ 1

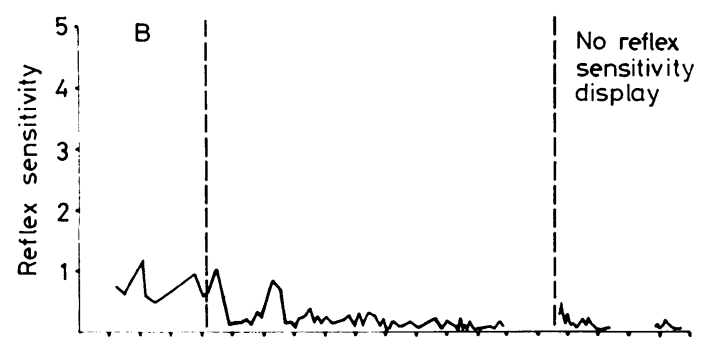

(c) SPAS SUBJ 3

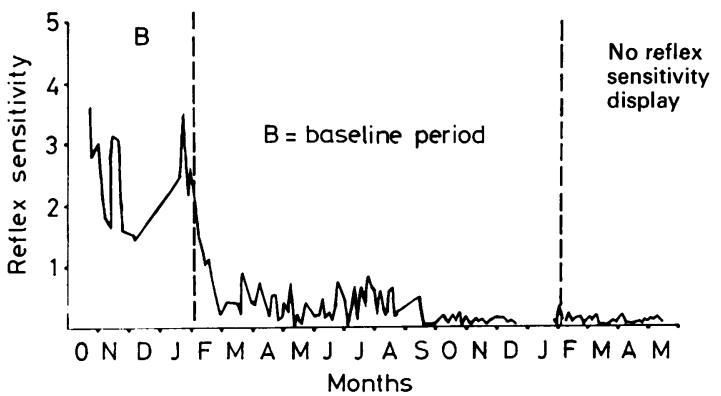

(b) ATH SUBJ 2

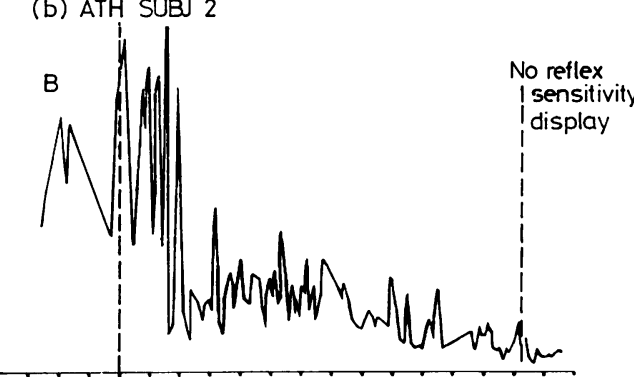

(d) SPAS SUBJ 4

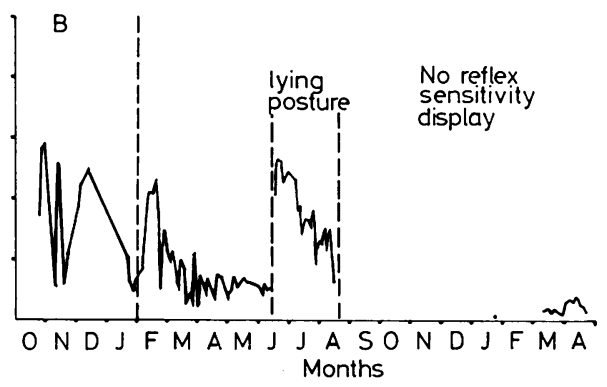

Fig 5 Graph of one-minute-average resting tonic stretch reflex sensitivity scores in arbitrary units plotted against days of training for (a) athetotic subject 1, (b) athetotic subject 2, (c) spastic subject 3, (d) spastic subject 4 showing effect of change from a sitting to a lying posture.

tonic stretch reflex sensitivity in the left arm independently of contraction level without any training on this arm.

After reflex training, all subjects were able to suppress resting tonic stretch reflex responses and regulate action tonic stretch reflex sensitivity two or three-fold independently of contraction level while the arm was oscillated back and forth through either 5 or 10 degrees peak to peak by the experimenter without the subject being able to see the reflex sensitivity display.

Error scores from the visual pursuit tracking tests show that functional control of arm movement in the athetotic subjects was inferior to that in the spastic subjects both before and after reflex training (table 1). Variance of arm movement correlated with stimulus marker movement was less (table 2) and variance of arm movement not correlated with stimulus marker movement was very much greater (table 3 ) in athetotic subjects than in spastic subjects both before and after reflex training.

Changes in functional control of arm movement associated with acquisition of ability to self-regulate spasm and spasticity and regulate action tonic stretch reflex sensitivity independently of contraction level were assessed by comparing tracking scores before reflex training with those after reflex training for each subject. Athetotic subject 1 improved tracking accuracy (table 1) and increased the amount of response movement correlated with stimulus marker movement (table 2), while the other athetotic subject showed only a small improvement and the spastic subjects showed negligible improvement on these scores. All subjects reduced the proportion of the total variance of arm movement not correlated with stimulus marker movement (table 4), as well as the variance of arm movement not correlated with stimulus marker movement (table 3). Reduction of inappropriate arm movement was greatest $(84 \%$ and $45 \%$ of pretraining variance) for the athetotic subjects who had excessive amounts of inappropriate arm movement before training. Improvement of tracking accuracy in the athetotic subjects was due primarily to this reduction in excessive level of inappropriate arm movement.

\section{Discussion}

Before training, cerebral palsied subjects were unable to suppress involuntary background activity (spasm and mobile spasm) and were unable to vary tonic stretch reflex sensitivity independently of 


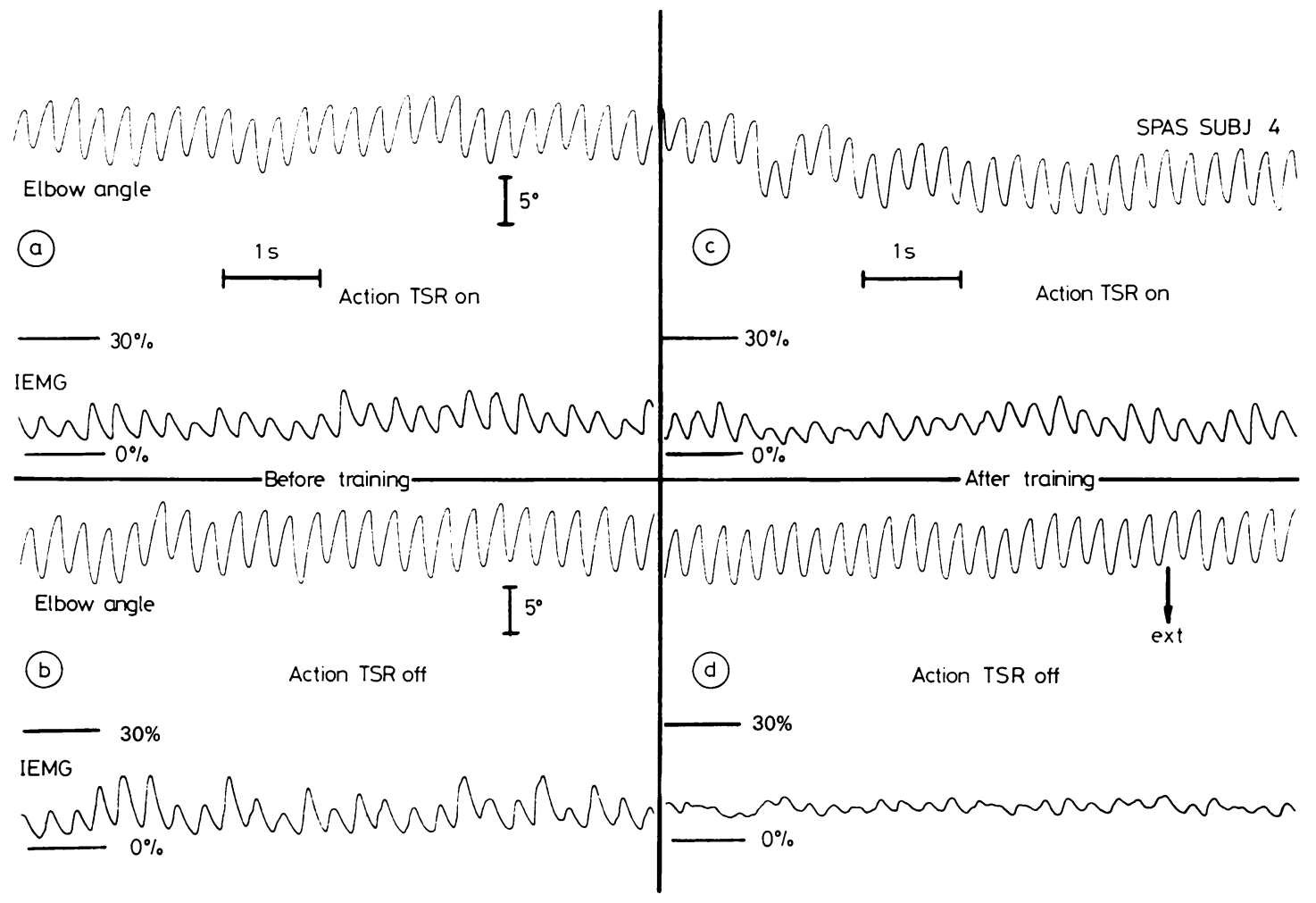

Fig 6 Sections of polygraph tracings from spastic subject 4 showing elbow-angle and IEMG signals while subject was attempting to sustain a voluntary contraction at $10 \%$ of maximum and either increase reflex sensitivity (action tonic stretch reflex on) or decrease reflex sensitivity (action tonic stretch reflex off). (a) Action tonic stretch reflex on before reflex training, (b) action tonic stretch reflex off before reflex training, (c) action tonic stretch reflex on after 6 months reflex training, (d) action tonic stretch reflex off after 6 months reflex training. Calibration bars as in fig 3.

contraction level. After training, they were able to suppress both involuntary activity and resting tonic stretch reflex responses and vary action tonic stretch reflex sensitivity two or three-fold independently of voluntary contraction level. In other words, cerebral palsied subjects learned to selfregulate spasm and spasticity at the elbow and to regulate action tonic stretch reflex sensitivity independently of contraction level. This ability was disrupted in one of the subjects by changing from a sitting to a lying posture and was then relearned in the lying posture. This indicates that the skill might be posture dependent and suggests that subjects should be trained in a variety of postures. A positive finding was that ability to regulate action tonic stretch reflex sensitivity could be transferred from the trained arm to the untrained arm without further training.

The resting tonic stretch reflex response in patients with spinal spasticity and hemiplegic spasticity consists of a burst of EMG activity at one $3^{* *}$ point in the stretching cycle with negligible EMG activity throughout the remainder of the stretching cycle. $^{3} 7$ This is very different from the resting tonic stretch reflex response described above in cerebral palsy, which was only present when there was a sustained level of involuntary background activity and was evoked by a relatively small angular displacement at the elbow. The background EMG activity was modulated by the sinusoidal perturbation applicd to the elbow-angle so that the IEMG also varied sinusoidally and followed the perturbation through the full stretching cycle. The magnitude of the resting tonic stretch reflex response was larger and the timing of the response was different ${ }^{4}$ from that reported previously in spinal spasticity ${ }^{3}$ and hemiplegic spasticity. ${ }^{7}$ The resting tonic stretch reflex response in cerebral palsy when involuntary background activity was present was similar in magnitude and waveform to the action tonic stretch reflex response with the same level of background activity sustained voluntarily. This 
(a) ATH SUBJ 1

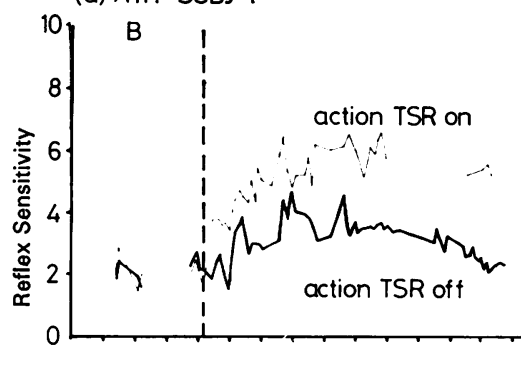

(b) ATH SUBJ 2

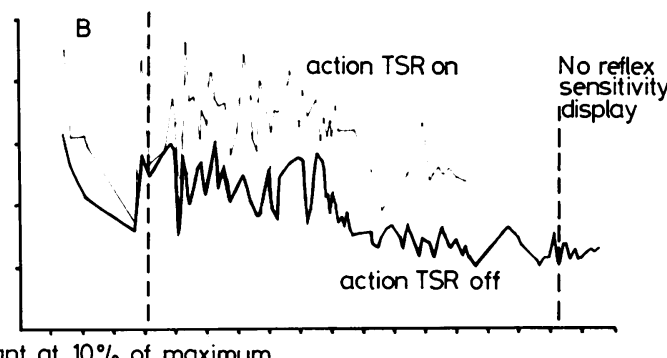

(c) SPAS SUBJ 3

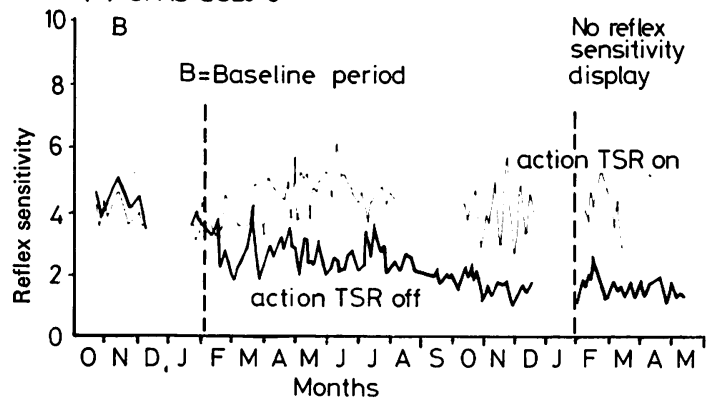

(d) SPAS SUBJ 4

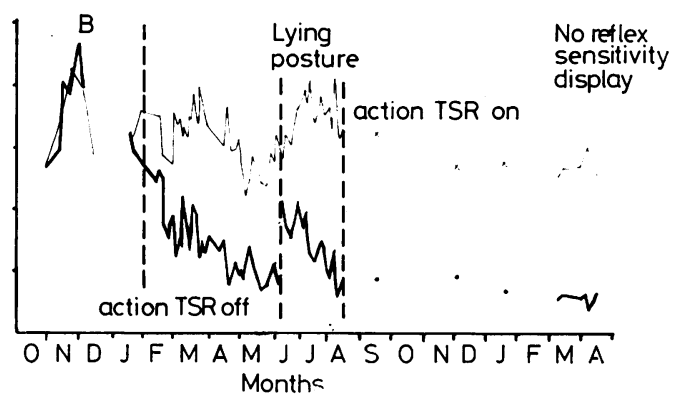

Fig 7 Graphs of one-minute-average action tonic stretch reflex sensitivity scores in arbitrary units plotted against days of reflex training while the subject maintained a constant contraction level at $10 \%$ of maximum and attempted either to increase reflex sensitivity (action tonic stretch reflex on) or decrease reflex sensitivity (action tonic stretch reflex off). (a) athetotic subject 1, (b) athetotic subject 2, (c) spastic subject 3, (d) spastic subject 4 showing effect of changing from a sitting to a lying posture.

suggests that, in cerebral palsy, spasticity assessed clinically when the patient is asked to relax may be caused in some patients by an abnormal resting tonic stretch reflex of the type observed in spinal spasticity and hemiplegic spasticity, ${ }^{3}{ }^{7}$ or it may be caused by an abnormal action tonic stretch reflex brought into play in association with involuntary background activity. The resting tonic stretch reflex almost certainly involves different reflex pathways from those involved in action tonic stretch reflex transmission and resting tonic stretch reflex and action tonic stretch reflex responses are influenced differentially by drugs such as phenoxybenzamine. ${ }^{4}$ Thus the reflex pathophysiology underlying spasticity assessed clinically will be different depending on whether or not the cerebral palsied patient is able to suppress involuntary activity when instructed to relax. Nevertheless, throughout this report the term "resting tonic stretch reflex" is used to refer to the tonic stretch reflex measured when the subject is attempting to reduce contraction level to zero.

During reflex training, the ability of cerebral palsied subjects to reduce resting tonic stretch reflex sensitivity and their ability to suppress involuntary background activity, varied together. A simple explanation for this finding is that they learned to inhibit alpha motoneurones. During reflex training, however, the main emphasis was not placed on teaching subjects to suppress involuntary activity or resting tonic stretch reflex sensitivity but on teaching subjects to regulate action tonic stretch reflex sensitivity independently of contraction level. This ability cannot be explained by inhibition of alpha motoneurones. A more likely explanation is that subjects learned to excite alpha motoneurones to maintain a voluntary contraction at the same time as they inhibited interneurones mediating action tonic stretch reflex transmission.

Comparison of visual pursuit tracking performance before and after reflex training shows that all subjects learned to reduce the amount of inappropriate arm movement and this reduction was most noticeable in the athetotic subjects who had excessive amounts of inappropriate movement initially. Reduction of excessive inappropriate movement (presumably due to dystonic posturing, spasm and 


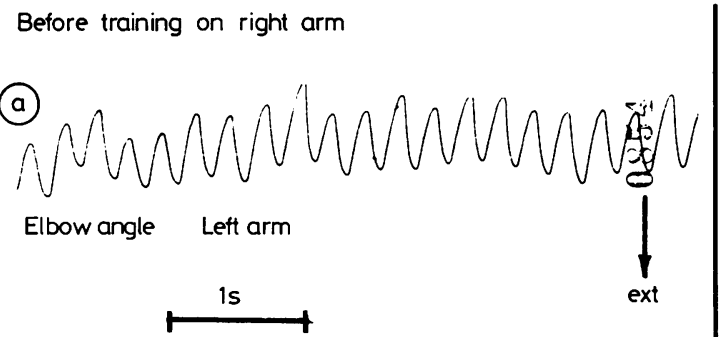

After training on right arm

(c)

SPAS SUBJ 4
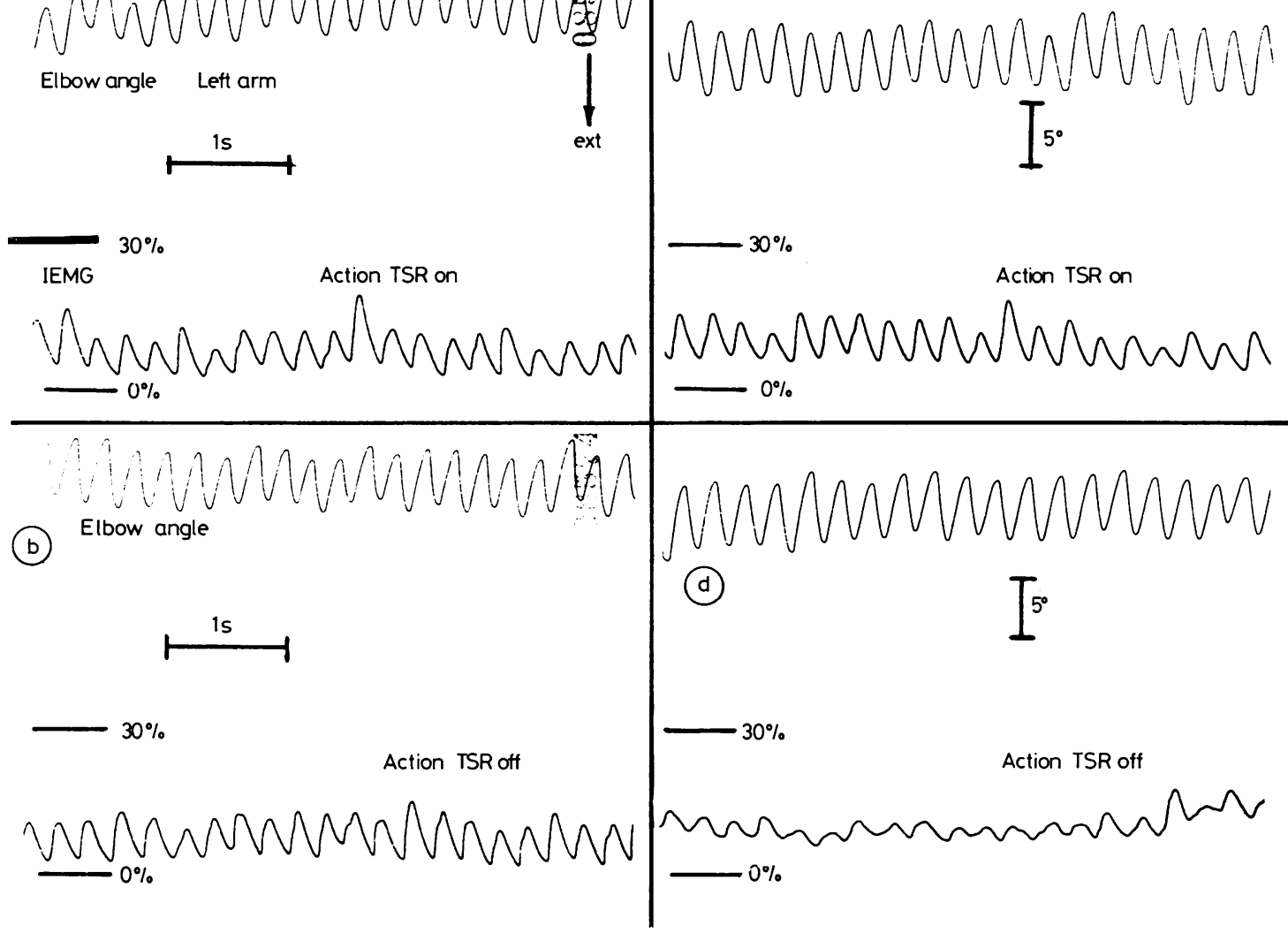

Fig 8 Sections of polygraph tracings showing elbow-angle and IEMG from the untrained left arm of spastic subject 4 attempting to maintain a constant contraction at $10 \%$ of maximum while either increasing reflex sensitivity (action tonic stretch reflex on) or decreasing reflex sensitivity (action tonic stretch reflex off). The subject could not see the reflex sensitivity display and had received no previous training on the left arm but had completed 6 months training on the right arm. (a) Action tonic stretch reflex on before training on right arm, (b) action tonic stretch reflex off before training on right arm, (c) action tonic stretch reflex on after 6 months training on right arm, (d) action tonic stretch reflex off after 6 months training on right arm. Calibration bars as in fig 3.

Table 1 Relative RMS error scores from the visual pursuit tracking tests

\begin{tabular}{|c|c|c|c|c|c|c|c|}
\hline \multicolumn{2}{|c|}{ Ath subj 1} & \multicolumn{2}{|c|}{ Ath subj 2} & \multicolumn{2}{|c|}{ Spas subj 3} & \multicolumn{2}{|c|}{ Spas subj 4} \\
\hline Before & After & Before & After & Before & After & Before & After \\
\hline $222 \%$ & $86 \%$ & $108 \%$ & $99 \%$ & $70 \%$ & $68 \%$ & $68 \%$ & $64 \%$ \\
\hline
\end{tabular}

Table 2 Overall gains from visual pursuit tracking tests

\begin{tabular}{|c|c|c|c|c|c|c|c|}
\hline \multicolumn{2}{|c|}{ Ath subj 1} & \multicolumn{2}{|c|}{ Ath subj 2} & \multicolumn{2}{|c|}{ Spas subj 3} & \multicolumn{2}{|c|}{ Spas subj 4} \\
\hline Before & After & Before & After & Before & After & Before & After \\
\hline 0.34 & 0.63 & 0.45 & 0.48 & $0 \cdot 76$ & 0.75 & 0.91 & 0.93 \\
\hline
\end{tabular}


Table 3 Variance of inappropriate arm movement in arbitrary units during visual pursuit tracking tests

\begin{tabular}{|c|c|c|c|c|c|c|c|}
\hline \multicolumn{2}{|c|}{ Ath subj 1} & \multicolumn{2}{|c|}{ Ath subj 2} & \multicolumn{2}{|c|}{ Spas subj 3} & \multicolumn{2}{|c|}{ Spas subj 4} \\
\hline Before & After & Before & After & Before & After & Before & After \\
\hline 52306 & 8108 & 9417 & 5317 & 3850 & 2898 & 4483 & 3916 \\
\hline
\end{tabular}

Table 4 Proportion of total arm movement variance not correlated with movement of the stimulus marker in the visual pursuit tracking tests

\begin{tabular}{|c|c|c|c|c|c|c|c|}
\hline \multicolumn{2}{|c|}{ Ath subj 1} & \multicolumn{2}{|c|}{ Ath subj 2} & \multicolumn{2}{|c|}{ Spas subj 3} & \multicolumn{2}{|c|}{ Spas subj 4} \\
\hline Before & After & Before & After & Before & After & Before & After \\
\hline 0.94 & 0.41 & 0.62 & 0.44 & 0.19 & 0.15 & $0 \cdot 16$ & $0 \cdot 14$ \\
\hline
\end{tabular}

athetoid movement) was accompanied by a reduction in error scores and an increase in overall gain in athetotic subject 1 . Nevertheless, functional control was still very poor in this subject relative to that in the spastic subjects. Ability to self-regulate spasm and spasticity was not accompanied by substantial improvement in functional control of arm movement in the other three subjects.

Dissociation between functional control of movement and ability to regulate spasm and spasticity supports the conclusion presented in a previous report ${ }^{8}$ that spasm and spasticity are not the primary cause of movement disability in cerebral palsy. This is not to say that spasm and spasticity do not disrupt voluntary movement. As illustrated by athetotic subject 1 in this study, excessive amounts of inappropriate movement and posturing prevent the subject from exerting even poor functional control. Nevertheless, when subjects acquired the ability to regulate spasm and spasticity and reduce inappropriate movement, the underlying functional control of movement remained very poor. This is consistent with the previous suggestion ${ }^{8}$ that movement disability in cerebral palsy results primarily from an inability to formulate and communicate appropriate motor commands to muscles rather than from disruption of voluntary movement by spasm and spasticity.

We wish to thank Mr Neil McLeod and members of the Board of the Spastic Centre of New South Wales for their support and for providing facilities within the Spastic Centre Research Unit at the University of New South Wales. We also wish to thank Professor JW Lance and Associate Professor D Burke for reading the manuscript and providing valuable suggestions.

\section{References}

${ }^{1}$ Kurland LT. Definitions of cerebral palsy and their role in epidemiologic research. Neurology (Minneap) 1957;7:641-54.

${ }^{2}$ Lance JW, McLeod JG. A Physiological Approach to Clinical Neurology. London: Butterworths, 1975.

${ }^{3}$ Burke D, Andrews CJ, Gillies JD. The reflex response to sinusoidal stretching in spastic man. Brain 1971; 94:455-70.

4 Neilson PD, Lance JW. Reflex transmission characteristics during voluntary activity in normal man and in patients with movement disorders. In: Desmedt JE, ed. Cerebral Motor Control in Man: Long Loop Mechanisms. Prog Clin Neurophysiol Vol 4, Basel: Karger, 1978:263-99.

${ }^{5}$ Neilson PD, Neilson MD. Influence of control-display compatibility on tracking behaviour. Quarterly Journal of Experimental Psychology 1980;32:125-35.

${ }^{6}$ Ashby P, Burke D. Stretch reflexes in the upper limb of spastic man. J Neurol Neurosurg Psychiatry 1971; 34:765-71.

${ }^{7}$ Milner-Brown HS, Penn RD. Pathophysiological mechanisms in cerebral palsy. $J$ Neurol Neurosurg Psychiatry 1979;42:606-18.

${ }^{8}$ Neilson PD, O'Dwyer NJ. Pathophysiology of dysarthria in cerebral palsy. $J$ Neurol Neurosurg Psychiatry. $1981 ; 44: 1013-9$. 